\title{
Silent gonadotroph pituitary neuroendocrine tumor in a patient with tuberous sclerosis complex: evaluation of a possible molecular link
}

\author{
Daniela Regazzo', Marina Paola Gardiman², Marily Theodoropoulou33, Carla Scaroni1, Gianluca Occhi4,* and \\ Filippo Ceccato1,*
}

\author{
1Department of Medicine DIMED, Endocrinology Unit, University Hospital of Padova, Padova, Italy, 2Department of \\ Medicine DIMED, Surgical Pathology \& Cytopathology Unit, University Hospital of Padova, Padova, Italy, \\ ${ }_{3}^{3}$ Medizinische Klinik und Poliklinik IV, Klinikum der Universität München, Ludwig-Maximilians-Universität München, \\ Munich, Germany, and ${ }^{4}$ Department of Biology, University of Padova, Padova, Italy \\ *(G Occhi and F Ceccato contributed equally to this work)

Correspondence should be addressed to G Occhi

Email

gianluca.occhi@unipd.it

\section{Summary}

Tuberous sclerosis complex (TSC) is an autosomal dominant multisystem hereditary cutaneous condition, characterized by multiple hamartomas. In rare cases, pituitary neuroendocrine tumors (PitNETs) have been described in patients with TSC, but the causal relationship between these two diseases is still under debate. TSC is mostly caused by mutations of two tumor suppressor genes, encoding for hamartin (TSC1) and tuberin (TSC2), controlling cell growth and proliferation. Here, we present the case of a 62-year-old Caucasian woman with TSC and a silent gonadotroph PitNET with suprasellar extension, treated with transsphenoidal endoscopic neurosurgery with complete resection. Therapeutic approaches based on mTOR signaling (i.e. everolimus) have been successfully used in patients with TSC and tested in non-functioning PitNET cellular models with promising results. Here, we observed a reduction of cell viability after an in vitro treatment of PitNET's derived primary cells with everolimus. TSC analysis retrieved no disease-associated variants with the exception of the heterozygous intronic variant c.4006-71C $>$ T found in TSC2: the computational tools predicted a gain of a new splice site with consequent intron retention, not confirmed by an in vitro analysis of patient's lymphocyte-derived RNA. Further analyses are therefore needed to provide insights on the possible mechanisms involving the hamartin-tuberin complex in the pathogenesis of pituitary adenomas. However, our data further support previous observations of an antiproliferative effect of everolimus on PitNET.

\section{Learning points:}

- Pituitary neuroendocrine tumors (PitNET) in patients with tuberous sclerosis complex (TSC) are rare: only few cases have been reported in literature.

- Therapeutic approach related to mTOR signaling, such as everolimus, may be used in some patients with PitNETs as well as those with TSC.

- We reported a woman with both non-secreting PitNET and TSC; PitNET was surgically removed and classified as a silent gonadotroph tumor.

- Everolimus treatment in PitNET's-derived primary cells revealed a significant decrease in cell viability.

- Considering our case and available evidence, it is still unclear whether a PitNET is a part of TSC or just a coincidental tumor. 


\section{Background}

Tuberous sclerosis complex (TSC) is an autosomal dominant multisystem hereditary cutaneous condition, characterized by multiple hamartomas that can be associated to endocrine system alterations $(1,2)$. TSC is mostly caused by mutations of two tumor suppressor genes TSC1 and TSC2, encoding for hamartin and tuberin, respectively. In normal cells, hamartin and tuberin form a molecular complex involved in intracellular signaling pathways controlling cell growth and proliferation, including the mammalian target of rapamycin (mTOR) cascade. Therapeutic approach related to mTOR signaling, such as everolimus, may be used in some patients $(1,2)$.

In nearly $10 \%$ of cases mutations are mosaic or intronic and a comprehensive genotype-phenotype correlation is still debatable. About two thirds of TSC cases are sporadic reflecting a high spontaneous mutation rate in these genes (3).

Some reports addressing PitNET in TSC patients suggest the possible involvement of pituitary gland alterations in the pathological process of TSC (4). The development of pituitary tumors by nearly half of adult Eker rats - i.e. spontaneous germline TSC2 mutants further supports this possible association (5). Up to now, however, no molecular evaluation of PitNET in patients with TSC has been performed to establish the weight of this link and whether PitNET should be considered a clinical manifestation of TSC is far from being clearly understood.

\section{Case presentation}

Here we present the case of a 62-year-old Caucasian woman whose diagnosis of TSC was made after the recognition of cognitive disability and neurobehavioral abnormalities of her only 32-year-old son, developed since childhood. She presented the typical TSC brain lesions at MRI (i.e. cortical tuber and right retinal hamartoma) without other typical TSC-related manifestations (e.g. renal, pulmonary, cardiac or skin lesions) and no family history of TSC. During the last 20 years she refused medical care, but in April 2012, she complained of headache: MRI scan showed a PitNET (size: $20 \times 18 \times 16 \mathrm{~mm}$ ) with suprasellar extension, bilateral cavernous sinus invasion, shortened pituitary stalk and left optic nerve compression (Fig. 1A). Clinically, she had central obesity, moon face, congenital left blindness and a normal right visual field. A hormonal study revealed
A

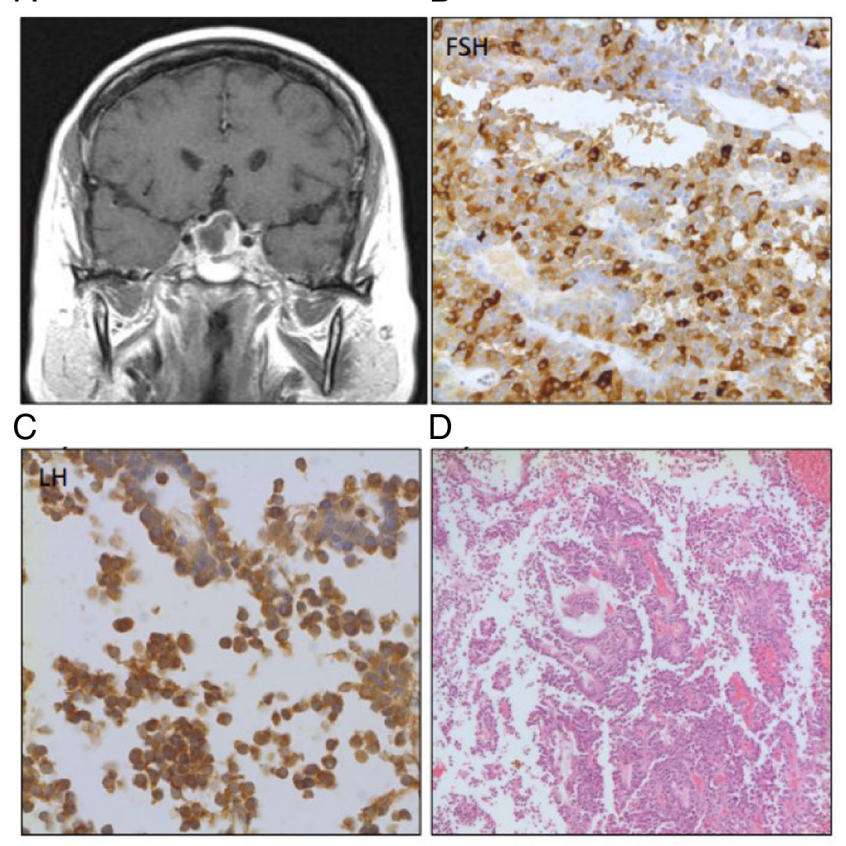

Figure 1

(A) Brain T1-weighted MRI after gadolinium injection: pituitary macroadenoma with suprasellar extension and bilateral cavernous sinus invasion; Immunohistochemical analysis of (B) FSH (20x magnification) and (C) LH (40x magnification) expression in the tissue slice of the silent gonadotroph PitNET (D) and standard hematoxylin-eosin staining.

normal thyroid and adrenal function, low gonadotropins (LH: $3.5 \mathrm{U} / \mathrm{L}$, range: 11-61; FSH: $13.5 \mathrm{U} / \mathrm{L}$, range: $35-150)$ and elevated prolactin $(75.7-74-79 \mu \mathrm{g} / \mathrm{L}$, range: $5-25$, probably due to shortened pituitary stalk). After 4 months, she underwent transsphenoidal endoscopic neurosurgery with complete tumor resection. Histologic evaluation demonstrated a uniform PAS-positive basophilic adenoma with low proliferation index (MIB-1: $<3 \%$ ). Immunohistochemistry was positive for FSH and LH (Fig. 1B and C) and negative for ACTH, GH, prolactin and TSH. Clinical and histological findings were thus consistent with a silent gonadotroph PitNET.

\section{Investigation}

Neurosurgery is the first treatment choice for PitNET (6), especially for those non-functioning, which are still orphans of medical therapy. Reduction of cell viability as a consequence of an increased apoptosis has been reported after an in vitro treatment of non-secreting PitNET with everolimus and its possible use in patients has been suggested (7). Given the therapeutic potential of everolimus in TSC (1) and PitNETs (7) - both conditions 
A

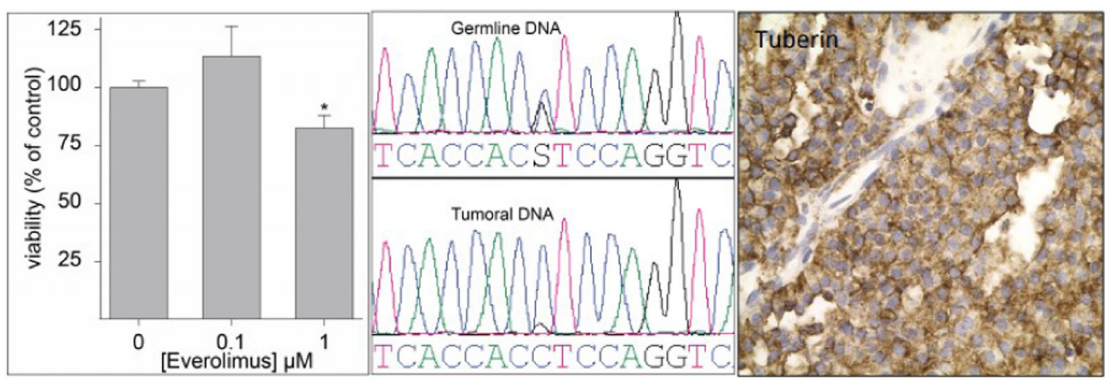

Figure 2

(A) Effects of everolimus treatment on pituitary tumor primary cells viability; (B)

Electropherograms centered on the c.4006-71C>T variant both in germline (upper panel) and tumoral (lower panel) DNA; (C)

immunohistochemical analysis of Tuberin expression (40x magnification) in the tissue slice of the silent gonadotroph PitNET (D93F12, XP Rabbit mAb of Cell Signaling at 1:100 dilution after microwave antigen retrieval in $10 \mathrm{mM}$ citrate $\mathrm{pH6.0)}$. present in our patient - primary cells derived from her pituitary adenoma were cultured in vitro (5000 cell/well in a 96 wells plate) and treated with everolimus (0.1 and $1 \mu \mathrm{M}$, kindly provided by Novartis) for evaluating its pharmacological effect. After 72-h treatment, the conversion of the tetrazolium dye MTT (Sigma-Aldrich) to formazan was ascertained following manufacturer protocol. After media removal and the addition of DMSO, the solution's absorbance was measured at $550 \mathrm{~nm}$ background subtraction set at $620 \mathrm{~nm}$ - with a microplate reader (Victor3 V 1420 Multilabel 206 Counter, Perkin Elmer). Treatment with $1 \mu \mathrm{M}$ induced a significant $20 \%$ decrease in cell viability $(P<0.05)$, confirming previous reported data (7) (Fig. 2A).

To search for the TSC disease-causative variant - and possibly associate it with pituitary adenoma development - patient's germline DNA was isolated from the peripheral blood and the genomic analysis of the entire TSC1 (NM_000368.4) and TSC2 (NM_000548.3) coding sequence and intronic boundaries was performed. The analysis retrieved no disease-associated variants with the exception of the heterozygous intronic variant c.4006-71C $>$ T found in TSC2 (Fig. 2B) and not present either in dbSNP or in ExAC databases. The computational tools MutationTaster and Human Splicing Finder both predicted a gain of a new splice site with consequent intron retention that was, however, not confirmed by an in vitro analysis of patient's lymphocyte-derived RNA. In addition, molecular analysis on archived paraffinembedded pituitary tumoral tissues failed to identify both loss of heterozygosity in TSC2 locus (Fig. 2B) and protein expression reduction (Fig. 2C), as expected if this would have been a loss-of-function mutation involved in the pathogenesis of this silent gonadotroph PitNET.

\section{Treatment}

We described the treatment in the intervention paragraph.

\section{Outcome and follow-up}

Pituitary imaging (with magnetic resonance) has been performed yearly, without evidence of a recurrent pituitary adenoma. The last visit was performed in May 2018.

\section{Discussion}

As far as we know, this is the first attempt to evaluate by a molecular approach the involvement of a TSC-related gene variant in the pathogenesis of the PitNET in a TSC patient. Considering the c.4006-71C $>\mathrm{T}$ variant causative for TSC - that is reasonable given the frequency of intronic mutations in TSC (i.e. 5\%) (3) and its absence in the normal population - we could exclude the involvement of pituitary gland alterations in the pathological process of TSC, at least in this patient. On the other hand, however, until functional data on this variant has not been gathered, we cannot exclude possible alternative genetic mechanisms causative of TSC including mosaic mutations in TSC1 regulatory regions - the mosaicism would easily explain the mild phenotype of our patient (3) - or an additional TSC causative gene. Nearly $10-15 \%$ of TSC patients lack, indeed, a conclusive molecular diagnosis (1, 2 ). Although relevant, our data are thus not conclusive for establishing unequivocally the causal nature of the association between TSC and PitNET that might only be a coincidence due to the relative high prevalence of pituitary tumors in the general population (8).

Although everolimus has been successfully tested in non-functioning and growth hormone-secreting PitNET cellular models $(7,9,10)$, very few data are available on its use in PitNET patients. Everolimus has indeed been used for treating only two patients with pituitary carcinomas resistant to repeated resections, radiations and combined treatment with chemotherapeutic agents i.e. Temozolomide and Capecitabine - with limited effects $(11,12)$. Our data corroborate the efficacy of everolimus in reducing cell viability in non-secreting PitNET and 
support the need of further studies for demonstrating its efficacy and safety for treating this group of tumors.

\section{Declaration of interest}

Filippo Ceccato: PhD grant founded by Novartis. All other authors declare that they have no conflicts of interest that might be perceived as influencing the impartiality of the reported research.

\section{Funding}

This work did not receive any specific grant from any funding agency in the public, commercial or not-for-profit sector.

\section{Patient consent}

Written informed consent for publication of their clinical details and/ or clinical images was obtained from the patient and is available for reviewers.

\section{Author contribution statement}

Daniela Regazzo, Marina Paola Gardiman, Marily Theodoropoulou performed the cell culture, in vitro study with everolimus, pituitary immunohistochemistry. Filippo Ceccato and Carla Scaroni were directly involved in the patient's clinical history and follow-up. Gianluca Occhi and Filippo Ceccato wrote the manuscript.

\section{References}

1 Orlova KA \& Crino PB. The tuberous sclerosis complex. Annals of the New York Academy of Sciences 20101184 87-105. (https://doi. org/10.1111/j.1749-6632.2009.05117.x)

2 Curatolo P, Bombardieri R \& Jozwiak S. Tuberous sclerosis. Lancet 2008372 657-668. (https://doi.org/ 10.1016/S0140-6736(08)61279-9)

3 Tyburczy ME, Dies KA, Glass J, Camposano S, Chekaluk Y, Thorner AR, Lin L, Krueger D, Franz DN, Thiele E, et al. Mosaic and intronic mutations in TSC1/TSC2 explain the majority of TSC patients with no mutation identified by conventional testing. PLoS Genetics 201511 1-17. (https://doi.org/10.1371/journal. pgen.1005637)

4 Dworakowska D \& Grossman AB. Are neuroendocrine tumours a feature of tuberous sclerosis? A systematic review. Endocrine-Related Cancer 200916 45-58. (https://doi.org/10.1677/ERC-08-0142)

5 Yeung RS, Katsetos CD \& Klein-Szanto A. Subependymal astrocytic hamartomas in the Eker rat model of tuberous sclerosis. American Journal of Pathology 1997151 1477-1486.

6 Chanson P, Raverot G, Castinetti F, Cortet-Rudelli C, Galland F, Salenave S \& French Endocrinology Society non-functioning pituitary adenoma work-group. Management of clinically nonfunctioning pituitary adenoma. Annales d'Endocrinologie 201576 239-247. (https://doi.org/10.1016/j.ando.2015.04.002)

7 Zatelli MC, Minoia M, Filieri C, Tagliati F, Buratto M, Ambrosio MR, Lapparelli M, Scanarini M \& Degli Uberti EC. Effect of everolimus on cell viability in nonfunctioning pituitary adenomas. Journal of Clinical Endocrinology and Metabolism 201095 968-976. (https://doi org/10.1210/jc.2009-1641)

8 Daly AF, Rixhon M, Adam C, Dempegioti A, Tichomirowa MA $\&$ Beckers A. High prevalence of pituitary adenomas: a crosssectional study in the province of Liège, Belgium. Journal of Clinical Endocrinology and Metabolism 200691 4769-4775. (https://doi. org/10.1210/jc.2006-1668)

9 Jalali S, Monsalves E, Tateno T \& Zadeh G. Role of mTOR inhibitors in growth hormone-producing pituitary adenomas harboring different FGFR4 genotypes. Endocrinology 2016157 3577-3587. (https://doi.org/10.1210/en.2016-1028)

10 Di Pasquale C, Gentilin E, Falletta S, Bellio M, Buratto M, Degli Uberti E \& Chiara Zatelli M. PI3K/Akt/mTOR pathway involvement in regulating growth hormone secretion in a rat pituitary adenoma cell line. Endocrine 201860 308-316. (https://doi.org/10.1007/ s12020-017-1432-0)

11 Jouanneau E, Wierinckx A, Ducray F, Favrel V, Borson-Chazot F, Honnorat J, Trouillas J \& Raverot G. New targeted therapies in pituitary carcinoma resistant to temozolomide. Pituitary 201215 37-43. (https://doi.org/10.1007/s11102-011-0341-0)

12 Donovan LE, Arnal AV, Wang S-H \& Odia Y. Widely metastatic atypical pituitary adenoma with mTOR pathway STK11(F298L) mutation treated with everolimus therapy. CNS Oncology 20165 203-209. (https://doi.org/10.2217/cns-2016-0011) 